\title{
Experience of Living and Coping with Spinal Cord Disability due to Road Traffic Injuries: A Phenomenological Study Running title: Living and Coping with Spinal Cord Disability
}

Mina Hashemiparast ( $\nabla$ minahashemiparast@gmail.com )

Zanjan Univercity of Medical Sciences

Hajieh Sheydaei

Maragheh University of Medical Sciences

Maryam Gharacheh

Iran University of Medical Sciences

Vijay Kumar Chattu

University of Toronto

\section{Research Article}

Keywords: Disability, Spinal cord injuries, Traffic accidents, Coping, Adaptation, Phenomenological research

Posted Date: September 27th, 2021

DOl: https://doi.org/10.21203/rs.3.rs-918239/v1

License: (1) This work is licensed under a Creative Commons Attribution 4.0 International License.

Read Full License 


\section{Abstract}

Background: Globally, people living with spinal cord disability experience more limitations in an individual and social life. In many cases, this leads to complex psychological and social problems that may also affect the adaptation to the conditions. The aim of the study was to explore the experience of living and coping with disability in people with spinal cord disability due to road traffic accidents in Iran's cultural context.

Methods: This is a qualitative study with a phenomenological approach on ten Iranian people with spinal cord disability due to road traffic injuries. Data collection and analysis were performed from September to March 2019. Data were collected by individual, face-to-face in-depth interviews, and the experience of living with disability and adaptation strategies were explored. Van Manen's methodical activities were used to guide the study's process.

Results: 'victim of destiny' was the main theme extracted from three themes and nine sub-themes. The disabled people viewed life as a prison that destiny had ordained for them and trapped them in the fences of isolation, anger, regret, anxiety, sorrow, pity, and futility such that they require assistance from others as dependent individuals. Religious recourse, satisfaction with God's expediency, and change of life values were the participants' coping strategies to adjust to their difficulties.

Conclusions: The study clarified the permanent constraints, needs, barriers and adaptation strategies of disabled people.

\section{Introduction}

Disability is a devastating condition that affects all aspects of physical, psychological and social health (1). Disabled people experience more limitations in an individual and social life (2).

Among disabilities, spinal cord injury (SCl) causes more limitations in motor, sensory or autonomic functions (3). Every year, more than tens of millions of people are injured on the world's roads and a significant proportion of disabilities are caused by road traffic crashes (4) (5)

It is reported that spinal cord injuries and mobility-related disability due to road traffic crashes are $14 \%$ more than other causes (6). This may be because, in non-fatal injuries, the lower limbs and spinal cord are most affected (7). Hence, people with road traffic disabilities experience more dependence and limitations in personal and social activities (8).

A study in Spain (2015) revealed disability due to road traffic injuries is associated with more need for medical care, social support, and significant changes in economic activities (6). It was documented in a qualitative study that survivors of road traffic accidents pointed to supportive needs, seek more information, and highlighted functional limitations (9). 
These limitations are more complicated for Iranian disabled people because in Iran, despite the government's efforts in recent years, disabled people still have poor access to health and rehabilitation services, education, employment and transportation, and public buildings (10).

Also, disability is a stressful situation that causes significant changes in all aspects of disabled individual's life. This situation may facilitate the occurrence of depressive incidence and may also affect the adaptation to the conditions (11) (12). Hence disabled individuals have to deal with the limitations of disability and have to cope with depression and stress due to disability.

Coping is "the methods that a person undertakes to deal with stressors"(13). The coping strategies that disabled persons use to cope with disability-related problems are diverse depending on the personality traits and socio-cultural context (14).

Hearing these people's voice and recognizing adaptation strategies is important to enable disabled people to promote acceptance, develop coping methods, and return to life. Therefore, this study aims to explore the experience of living and coping with disability in people with spinal cord disability due to road traffic accidents in Iran's cultural context.

\section{Methods}

\subsection{Study design \& participants}

This study used a phenomenological approach to explore the experiences of living and coping with disability in people with spinal cord disability due to road traffic injuries. The main research question was: How do people with spinal cord disability due to road traffic injuries describe and interpret their living experiences and cope with a disability?

Van Manen's six methodical activities were used to guide the study's process (Table 1$)(15,16)$. 
Table 1

Summary of van Manen's qualitative phenomenological approach

\section{Six methodical The researcher's activities activities proposed by van \\ Manen (1990)}

Turning to the nature of lived experience
The activities were directed towards thinking about, understanding, interpreting, and discoursing with people with spinal cord disability due to road traffic injuries, formulating the phenomenological question: What is it like to experience disability?

Investigating experience as we live it

Reflecting on essential themes

Experiential data were collected using various activities, including observation, prolonged engagement with the participants, phenomenological literature, conducting in-depth interviews, protocol writing, and journals.

Determine emerging themes that characterize the phenomenon through immersing in data by listening to the audio recordings, reading transcripts, and conducting thematic analysis through holistic, selective, and detailed approaches.

Writing transcripts, writing about concepts, sub-themes, and themes, writing to

Hermeneutic phenomenological writing

Maintaining a strong and oriented relation to lived experience

Balancing the research context by considering parts and whole create a phenomenological text by describing the phenomenon of disability in the art of writing and rewriting.

Discussing the themes concerning health science and keeping an orientated relation upon the lived experience of disability.
Moving between the transcripts, meaning units, concepts, sub-themes, and themes in relation to health science to understand the phenomenon of disability.

Participants were a purposive sample of disabled patients with an SCI due to road traffic injuries referred to the rehabilitation centers in East Azerbaijan, Iran. Purposeful sampling with the maximum variation in terms of age, gender, type of crash (car driver, pedestrian, and/or car's passenger), and duration of disability was used to select the participants. The details about the participants are presented in Table 2 . 
Table 2

Profile of the participants' characteristics $(n=10)$

\begin{tabular}{|llllll|}
\hline Participant & Gender & $\begin{array}{l}\text { Age } \\
\text { (year) }\end{array}$ & Marital status & $\begin{array}{l}\text { Duration of disability } \\
\text { (year) }\end{array}$ & Type of crash \\
\hline P1 & male & 21 & single & 17 & Pedestrian \\
\hline P2 & female & 31 & single & 6 & car's passenger \\
\hline P3 & male & 24 & single & 7 & car's passenger \\
\hline P4 & male & 64 & married & 37 & car's passenger \\
\hline P5 & female & 32 & married & 3 & car's passenger \\
\hline P6 & male & 43 & married & 17 & car's passenger \\
\hline P7 & male & 55 & married & 8 & car driver \\
\hline P8 & female & 20 & single & 6 (month) & car's passenger \\
\hline P9 & female & 33 & divorced & 4 & car's passenger \\
\hline P10 & female & 48 & single & 3 & Bus passenger \\
\hline
\end{tabular}

The spinal cord injury due to a road traffic accident and being able to communicate with the interviewer were the study inclusion criteria.

\subsection{Data collection}

Data collection and analysis were performed from September to March 2019. Data were collected by individual, face-to-face in-depth interviews. The interviews usually were begun with a general question: 'Could you please tell me about your experience of living with a disability?' The other questions asked by the interviewer were: 'What changes did you make in your life after disability?', 'How do you feel about your physical condition?', 'What is it like the experience of spinal cord disability for you?' and 'How did you cope with this condition?'.

Additionally, further explanations were also obtained based on descriptions of the participants and by asking complementary probing questions such as "explain your experience more please."

Interviews were conducted by a clinical psychologist (the second author, MSc) who was trained and familiar with the qualitative interview methods. This allowed us to support the interviewees who had negative emotions during the interview sessions due to their unpleasant experiences.

The interviewees determined the time of the interviews. Hence, each interview lasted 30-60 minutes till they stopped sharing their experiences. All interviews were performed in a private and convenient place for each participant at the rehabilitation center. Each interview was audio-recorded and transcribed 
verbatim for the first interpretation and further analysis before the next interview. The interviews were continued until the required richness, where no new theme or idea emerged.

\subsection{Data management and analysis}

The transcribed texts were analyzed using thematic analysis, including holistic, selective, and detailed approaches $(16,17)$. Through holistic approach, each transcript was read repeatedly to achieve a sense of the whole and create a phrase that grasped the meaning of the participant's whole experience. In the selective approach, the principal researcher (the first author) looked for phrases that appeared mainly essential about the studied phenomenon- living and coping with disability. These phrases were highlighted to recognize themes from the initial concepts. In the detailed approach, the researcher examined each statement of the transcripts to extract units of meaning. Then, the formulated meanings were interpreted and sub-themes were identified and grouped into themes. MAXQDA software version 10 was used to manage the textual data during the coding process.

\subsection{Trustworthiness}

This study applied the criteria suggested by Guba and Lincoln (18) to evaluate the data's rigor. The prolonged engagement with participants during the interview period helped establish trust and a deeper understanding of the participants' experiences. Triangulation of data collection methods was done using field notes and diaries. Peer debriefing was conducted to indicate our position toward the data and analysis. Hence, the research team checked the interview data and findings at each step of the study. The final themes were revised with the research team members and were interrelated and synthesized into a comprehensive phenomenon. Moreover, the transcripts of the interviews and interpretations were taken back to the participants to verify before conclusion.

All the steps were followed in the research process, and the researchers documented the participants' backgrounds to provide auditability and dependability of the data. The guidelines of consolidated criteria for reporting qualitative research (COREQ) were used for developing this manuscript.

\subsection{Ethical considerations}

The ethics committee of Maraghe university of Medical Sciences (MRGUMS) approved the study protocol (Approval ID: IR.MARAGHEHPHC.REC.1397.007). The study's aim and process were explained to the participants, and written informed consent was obtained.

\section{Results}

'Victim of destiny' was the main theme extracted from three themes and nine sub-themes that described the experiences of disabled people about their disability-related challenges. Moreover, religious recourse, satisfaction with God's expediency, and change of life values were the coping strategies the participants used to adjust to their difficulties (Table 3). 
Table 3

Summarizing the themes and sub- themes

\begin{tabular}{|c|c|c|}
\hline Main Theme & Theme & Sub- theme \\
\hline \multirow[t]{12}{*}{ Victim of destiny } & \multirow[t]{2}{*}{ Prison of fate } & - Surrender of fate \\
\hline & & - Divine test \\
\hline & \multirow[t]{5}{*}{ Living behind the fence } & -Prison of loneliness \\
\hline & & - Feeling of futility \\
\hline & & - Feeling of being a burden \\
\hline & & - Pathetic social interactions \\
\hline & & - Fear of the future \\
\hline & \multirow[t]{2}{*}{ Captive of restrictions } & - Physical restrictions \\
\hline & & - Need to support \\
\hline & \multirow[t]{3}{*}{ Copping strategies } & -Religious recourse \\
\hline & & -Satisfaction to the expediency of God \\
\hline & & -Change of life values \\
\hline
\end{tabular}

'Victim of destiny' refers to the conditions that participants inevitably encountered. Disabled people living with disabilities face numerous irreversible restrictions and get trapped in the fences of loneliness, frustration, regret, fear, grief, pity, and feeling of futility in their everyday life. Participants described themselves as dependent people that need others' help, so they view life as a prison that destiny had ordained for them and for which they have no option but a painful surrender.

\subsection{Prison of fate}

'Prison of fate' reflects a picture of the condition shaped by God's will. This situation has occurred out of the participants' will, so they had no choice except surrender. This theme extracted from two sub-themes as follows:

\subsubsection{Surrender to fate}

Participants believed disability was the fate predestined in their fortune and could not be escaped from it; therefore, they have to surrender it inevitably and continue living in this way. In explaining this idea, participant 1 said:

"... It's a destiny for us to be like that. Everyone has a destiny, so we should accept it." (p1) 
They considered themselves forced to continue living with this situation because they did not have the authority to change their fate. This can be understood from the expression of one of the participants who stated:

"... It happened for me, and there is nothing else I can do. I have to endure this situation because I'm alive and I have to live." (p2)

\subsubsection{Divine test}

Some participants considered disability an outcome of God's experiment to believe God tested them by making them suffer a disability. Although they were surrendered to the divine test, they did not consider themselves worthy of it, and complained of God to be stuck in such a disaster. One participant who had a spinal cord injury in childhood said:

"... I was a child when I had an accident, and I did not have any sin. It was not right. Why should God test me? I shouldn't have been punished?"( 1 1)

\subsection{Living behind the fence}

This theme refers to the experience of deep emotions such as feelings of loneliness, futility, being a burden to others, piety, and fear of the future. The participants perceived this situation as a fence that surrounded their life. This theme extracted from five sub-themes as follows:

\subsubsection{Prison of loneliness}

This sub-theme reflects a profound sense of loneliness that had enclosed the participants' life. Participants experienced loneliness in different ways, including lack of family support, neglect by others, and not being understood by citizens. Lack of family support was expressed in the participant's words as:

"...I heard from my parents that said "we cannot keep her. We have to give her to the welfare institute. It is not our duty to take care of her". It means that my family had abandoned me, and after that, I realized that I am so alone. " (p9)

Being ignored by others during the time was another bitter experience that fueled participants' feelings of loneliness:

"... Before the injury, the world was beautiful. Everyone was kind to me, but after that, the others were kind just for the first month. Even my sisters left me at the hospital. I knew that if my parents don't support me I must be alone for the rest of my life." (p3)

Not being understood by people and being ignored by citizens were other reasons that made the participants stuck in solitary confinement:

"... I'm annoyed with others. They do not understand my suffering well. They cannot sit-up in a wheelchair for a full day. Although people with disabilities have an ID card to not standing in the gas station queue, 


\subsubsection{Feeling of futility}

This sub-theme reflects the unpleasant feeling of inefficiency and uselessness that has shadowed all aspects of the participants' life. According to the participants, dependence on others in doing personal duties and lack of economic dynamics resulted in considering themselves worthless; so that some participants likened their lives to a parasite or a mobile fertilization machine:

".. I feel like a parasite. When I cannot bring a glass of water for myself, this life is completely worthless." (P2)

"... I used to have a job and income, but now I feel that I have become a useless entity, and I'm not able to do anything, just as a mobile fertilizer machine; completely useless."(P7)

\subsubsection{Feeling of being a burden to others}

Another bitter emotion that participants were struggling with it was the feeling of being a burden to others. Feeling of helplessness in doing personal tasks and constant dependence on others due to disability made them feel shameful. In support of this idea, some of the participants said:

"...It's so hard for me to have someone next to me all the time. It hurt my own life, and the life of others around me. Any other people involved in my tasks. I wish l'd died, and it would not be." (P5)

"I am not able to do anything by myself. If people do not help me, I will not do anything. I am dependent on my parents. I feel like I'm an extra burden." (P2)

\subsubsection{Pathetic social interactions}

Another unpleasant experience that the participants pointed out in their statements was the overwhelming concern of others perceived as pity and insult. Discomfort with the compassion of others was a feeling experienced by participants in various ways:

"... The teachers made upset me with their behavior. They got a score of twenty for me in the sport class. I wish they gave me a ball, letting me kick to the wall and get my real score. This behavior is considered insulting." (P1)

\subsubsection{Fear of the future}

The fear of losing the patron and caregiver created an ambiguous picture of the future for the participants. They had a great fear of being isolated and disabling to live in the future due to the limitations resulting from disabilities. They were constantly thinking about what future they would expect. Concern for the future was expressed in the participant's word as:

"...I'm always thinking about the future. If my wife dies, what shall I do? How can I live? When my children will get married, what will I do?"(p5) 
"... My greatest fear is that my parents will die one day. I do not know what I will do when they don't exist in the world." $(\mathrm{p} 2)$

Fear of remaining in a wheelchair for the rest of life, was another negative emotion about the future. This fear reflected their frustration about recovery. Most of the participants reported that they have no hope to recover:

"... I'm disappointed and assume that I cannot walk anymore. I can no longer back the ability to walk that I used to have. I have to stay on the wheelchair for the rest of my life "(p8)

\subsection{Captive of restrictions}

This theme points to the participants' suffering from the constraints of disability, which had faced them with physical challenges. On the other hand, physical restrictions caused them to need support from the government for acting in the community as a citizen. This theme extracted from two sub-themes as follows:

Physical problems caused by disabilities imposed restrictions on the participants' lives, leading feelings of helplessness and frustration. Not being able to do the job as before increased their restrictions. This perspective was described as follows:

"...Before the injury, I worked since the morning to the night, I had no problems, but after that, I, physically and sexually faced the problem. After the injury, everything was finished at once."(p6)

In this regard, one of the participants likened his life to a bird that is not able to fly due to restriction:

"... After the injury, I cannot play sports anymore. Before the injury, I played football and tennis. I cannot travel anymore. I lost my sense of sexual activities. My body is always in pain. Since the injury, I'm like a bird that is not able to fly."(p4)

\subsubsection{Need for support}

This sub-theme reflects the participants' needs for support from the government and custodians. The lack of facilities for disabled people had limited their presence in the community. Participants complained about inappropriate situation of streets and argued that urban furniture was not tailored to the needs of disabled people; so that one of the participants likened a minor height difference for healthy people to a tall summit for the disabled ones:

"...When I go out of the house, everything puts pressure on me. For example, a small height difference may not seem for a normal person, but it is as high as Everest Mountain for me." (p2)

"... This city does not consider disabled people. Nowhere has been adapted for the needs of disabled people; neither our pedestrian walks nor our offices are suitable for us." (p10)

\subsection{Coping strategies}


Coping strategies refer to the methods that help disabled people cope with the emotional distress caused by disability. Participants believed that religious recourse, accepting God's will's practicality, and change of life values made the suffering of disability more tolerable for them. These concepts explained below:

\subsubsection{Religious recourse}

According to the participants, spirituality was perceived as a stronger source of support in confronting such difficult situation. Recourse religious beliefs and practices such as praying, trust and taking refuge in God help them reduce their negative emotions. They believed that mentioning God and praying has positively affected their psychological state. In support of this idea one of the participants said:

"...I always engage with God in secret prayer. I seek refuge in God. God is very powerful. Whenever I am disappointed, I talk with God, I pray, and I believe God help me. It is not difficult for God to heal me." (p8)

\subsubsection{Satisfaction to the expediency of God}

The disabled people put their suffering into god will frameworks. They considered disability a destiny that occurred by God's will and wisdom so they have to surrender to divine providence:

"... Everything is not under the control of the human beings. This accident happened from God. No one can withstand the will of God. So I am satisfied with God's will."(p7)

\subsubsection{Change of life values}

Some participants believed that disability also had some valuable achievements which allowed them to better understand the meaning of life. They declared, overtime after they learned how to live with disability they achieved the goal of creation:

"... I used to think I came to this world just to be happy and to live, but now I understand the purpose of creation. I found God and realized that with divine tests, we could know ourselves and reach perfection and that is the perfection of God." (p6)

Moreover, experiencing life with a physical disability provided the individual opportunities to be patient in the face of life's difficulties:

"... Disability made me more patient with life's problems. Dealing with problems gave me experience, and I believe hardship is for all human beings, you just have to be patient." (p10)

\section{Discussion}

The study explored the experiences of living and coping with disability in people with spinal cord disability due to road traffic injuries. The findings revealed that, besides physical limitations, the people with spinal cord injury experienced various negative psychological and social consequences in their daily life and had adopted some coping strategies in accepting the process and adjusting to the hardships. 
In this study, "victim of destiny" was identified as the main theme that provides a picture of forced destiny in the participants' life and a fate outside of their will. They considered themselves as victims of fate and believed this situation was a destiny predetermined in their life caused by divine will and wisdom, so they had no options but to surrender.

Belief in destiny is a common phenomenon in the Iranian cultural context. According to Islam teachings, nothing happens outside of the divine will, so one tends to attribute all the life events, especially negative ones, to divine destiny. This belief leads to peace and hope in difficult circumstances (19).

Previous studies have also pointed to the belief in divine will among patients. Accepting what comes from God and destiny helped the patients in finding and keeping hope. The study of Baba Mohammadi et al. (2013) showed people with spinal cord injury attributed their disability to the divine destiny, which helped the patients to be able to adjust to the situation (20). Also, in a study on coping strategies for breast cancer in Iranian women, belief in divine will and the occurrence of God-given disease was a strategy that helped patients cope with the illness (21). The belief that all these come from God is vital in accepting the process and adjusting to the situations.

Besides, physical limitations of disability and loss of ability to do the job made participants feel ineffective and worthless. The dependence on others for performing personal duties had also led to the feeling of being a burden among the participants. It seems the feeling of being a burden to others is a common experience among people with physical-motor disabilities.

In other studies, inefficiency due to job loss and economic dependence has been described as an unpleasant experience after spinal cord injury. In the study of Sharifi et al., the sense of inferiority caused by being a burden to others and uselessness in the community was an experience that individuals with physical-motor disabilities also mentioned in their experiences (1).

Another problem commonly experienced by the participants was pathetic social interactions. The findings suggested pitiful look, and people's pathetic behavior led to negative and unpleasant emotions such as humility in participants. The unnecessary compassion and unwanted assistance or support provided to them were meant as commiseration by participants. It was unpleasant to the participants and; made them sad and upset because they expected people to interact with them like other citizens in social interactions.

Sense of pity and misplaced compassion of the community members toward people with spinal cord disability has also been emphasized in other studies. For instance, in a qualitative study from Ghana that explored lived experiences of people with spinal cord injury, participants expressed frustration that the society saw them as disabled people and treated them with sympathy (1).

In a study by Khanjani et al. (2019), sympathy was identified as one of the socio-cultural misconceptions and a deterrent to adjustment to the illness. It was also considered an annoying behavior for patients with spinal cord injury (22). 
In the Iranian cultural context, compassion for the underprivileged and disabled people is considered a noble behavior. Based on religious beliefs, people feel that they have some kind of spiritual insurance by helping disabled people and providing a sense of compassion. In fact, they believe paying attention to people with disabilities and providing them with voluntary assistance will reward them in the future. That is why they help these people regardless of the feelings of the disabled persons.

Besides, fear of the future was commonly experienced by the participants in various ways in their daily life. The fear of losing caregivers due to their dependence on others to perform daily duties made participants anxious and fearful. Moreover, the frustration with the recovery and the fear of being permanently dependent on others was an unpleasant feeling that participants had to deal with in their daily lives.

In line with the participants' experiences, people with spinal cord disabilities pointed to their dependence on others to meet their personal needs. They believed that they had to rely on others even for their daily care. Their most significant concern was the fear of their dependence on others (23).

Participants after the injury also experienced a sense of loneliness. Lack of support from the family, a sense of being rejected by friends and being ignored by others made the participants feel isolated and alone. According to the participants, lack of family support played an important role in the development of this unpleasant feeling. In contrast, in Iranian culture, the family is considered the most critical source of support for the patient in the face of the disease and its challenging situations (24).

Receiving support from family, particularly the significant persons, has been identified as a facilitator in reducing symptoms of depression among people with spinal cord injury and their return to life (25).

Lack of government support, the inefficiency of supportive organizations to provide appropriate services for disabled people, and lack of urban furniture tailored to the disabled people's conditions were other problems that participants noted in their experiences. Challenges related to social services delivery and neglecting the disability-friendly cities have also been reported in other studies as the shared experiences among disabled people (26).

The results obtained from this research regarding coping strategies against the disability have been raised to focus on the theme of religious/spiritual coping. In line with related research in other cultures, spirituality and religion were stated as essential elements of support in coping with stressful life events $(27,28)$. Hence, find religiously oriented mechanisms like satisfaction to God's practicality and accept what comes from act as a source of hopefulness and comfort.

\section{Conclusion}

This study revealed that spinal cord disability imposed irreversible restrictions on the participants' lives. As dependents, they needed support from others and being desperate to recover their lost power, they identify themselves as victims of destiny. Hence, social support, empowering disabled people to 
overcome limitations, and adopting religious recourse to overcome the problems can recover their mental health and help them return to a normal life. Moreover, given the unpleasant experiences of participants in social interactions, creating a socio-cultural context on dealing with people with disabilities is essential.

\section{Declarations}

\section{Acknowledgement}

The authors would like to acknowledge from participants for sharing their experiences in the study.

\section{Conflict of interest}

The authors have no conflict of interest to declare.

\section{Authors' Contribution}

$\mathrm{MH}$ and $\mathrm{MGH}$ provided a research proposal and final report. $\mathrm{HSH}$ have done data gathering. $\mathrm{MH}$ provided the initial draft of the manuscript. VK also participated in editing the document and delivering the final version. All the authors read and approved the final version of the manuscript.

\section{References}

1. Fuseini A-G, Aniteye P, Alhassan A. Beyond the Diagnosis: Lived Experiences of Persons with Spinal Cord Injury in a Selected Town in Ghana. Neurology research international. 2019;2019.

2. Kurawa SS. The impact of disability on self and society: An agenda for research on rehabilitation of disabled in Nigeria. Procedia-Social and Behavioral Sciences. 2010;5:1804-10.

3. Mtetwa L, Classen S, Van Niekerk L. The lived experience of drivers with a spinal cord injury: A qualitative inquiry. South African Journal of Occupational Therapy. 2016;46(3):55-62.

4. https://. Violence and Injury Prevention 2019.

5. WHO, https://www.who.int/violence_injury_prevention/disability/en/. Injury-related disability and rehabilitation https://www.who.int/violence_injury_prevention/disability/en/

6. https:// [cited 2019].

7. Palmera-Suárez R, López-Cuadrado T, Almazán-Isla J, Fernández-Cuenca R, Alcalde-Cabero E, Galan I. Disability related to road traffic crashes among adults in Spain. Gaceta sanitaria. 2015;29:43-8.

8. Malm S, Krafft M, Kullgren A, Ydenius A, Tingvall C. Risk of permanent medical impairment (RPMI) in road traffic accidents. Annals of advances in automotive medicine Association for the Advancement of Automotive Medicine Annual Scientific Conference. 2008;52:93-100.

9. Kellezi B, Coupland C, Morriss R, Beckett K, Joseph S, Barnes J, et al. The impact of psychological factors on recovery from injury: a multicentre cohort study. Soc Psychiatry Psychiatr Epidemiol. 2017;52(7):855-66. 
10. Sabet FP, Tabrizi KN, Khankeh HR, Saadat S, Abedi HA, Bastami A. Road traffic accident victims' experiences of return to normal life: A qualitative study. Iranian Red Crescent Medical Journal. 2016;18(4).

11. Soltani S, Takian A, SARI AA, Majdzadeh R, Kamali M. Financial Barriers to Access to Health Services for Adult People with Disability in Iran: The Challenges for Universal Health Coverage. Iranian Journal of Public Health. 2019;48(3):508.

12. Turk MA, McDermott S. Disability, stress, and health disparities. Disability and health journal. 2018;11(3):331-2.

13. Bramston P, Mioche C. Disability and stress: A study in perspectives. Journal of Intellectual and Developmental Disability. 2001;26(3):233-42.

14. Min LJ, Drury VB, Taylor BJ. The experiences of and meaning for women living and coping with type 2 diabetes: a systematic review of qualitative evidence. JBI Database of Systematic Reviews and Implementation Reports. 2012;10(47):2998-3047.

15. Kuo B. Culture's Consequences on Coping. Journal of Cross-cultural Psychology - J CROSS-CULT PSYCHOL. 2011;42:1084-100.

16. Van Manen M. Researching lived experience: Human science for an action sensitive pedagogy: Routledge; 2016.

17. Errasti-Ibarrondo B, Jordán JA, Díez-Del-Corral MP, Arantzamendi M. Conducting phenomenological research: Rationalizing the methods and rigour of the phenomenology of practice. Journal of advanced nursing. 2018;74(7):1723-34.

18. Philipsen B, Tondeur J, Pynoo B, Vanslambrouck S, Zhu C. Examining lived experiences in a professional development program for online teaching: A hermeneutic phenomenological approach. Australasian Journal of Educational Technology. 2019;35(5):46-59.

19. Guba EG, Lincoln YS. Fourth generation evaluation: Sage; 1989.

20. 19.. the Faculty of Literatures \& Humanities. 2004;541)55ロ):-.

21. Babamohamadi H, Negarandeh R. Important coping strategies used by individuals with spinal cord injury: A qualitative study. Journal of Qualitative Research in Health Sciences. 2013;2(1):90-100.

22. Taleghani F, Yekta ZP, Nasrabadi AN. Coping with breast cancer in newly diagnosed Iranian women. Journal of Advanced nursing. 2006;54(3):265-72.

23. Khanjani MS, Khankeh HR, Younesi SJ, Azkhosh M. The Main Factors Affecting the Acceptance and Adaptation With Spinal Cord Injury: A Qualitative Study. 2019.

24. Nikbakht A, Gomarverdi S, Bastami A, pashaii sabeti F, Bastami M, Sabaghieh Yaz MR. Spouses' experiences of living with patients with spinal cord injuries: a phenomenological study. Iranian Journal of Nursing Research. 2017;12(3):24-35.

25. Allahbakhshian A, Hasankhani H, Mohammadi E, Zamanzadeh V, Allahbakhshian M. Coping strategies used by patients after coronary angioplasty: a qualitative study. Iranian Journal of Nursing Research. 2015;10(1):42-55. 
26. Kalpakjian CZ, Houlihan B, Meade MA, Karana-Zebari D, Heinemann AW, Dijkers MP, et al. Marital status, marital transitions, well-being, and spinal cord injury: an examination of the effects of sex and time. Archives of physical medicine and rehabilitation. 2011;92(3):433-40.

27. Walker A. Disability and dependency: a challenge for the social services. Research Policy and Planning. 1983;1.

28. Psarra E, Kleftaras G. Adaptation to physical disabilities: The role of meaning in life and depression. 2013.

29. Hatun O, Yavuz-Birben F, Ince Z, Kalkan-Yeni G. The ticket to heaven: A spiritual resource for coping with disability. Spiritual Psychology and Counseling. 2016;1(2):209-35. 